\title{
整车室内道路模拟试验用载荷谱的编制方法研究*
}

\author{
于佳伟 ${ }^{1}$ 郑松林 ${ }^{1,2}$ 赵礼辉 ${ }^{1,2}$ 赵子顺 ${ }^{3}$
}

(1. 上海理工大学机械工程学院 上海 200093;

2. 上海理工大学机械工业汽车底盘零部件强度与可靠性评价重点实验室 上海 200093;

3. 浙江吉利汽车研究院有限公司 杭州 311228)

摘要: 室内道路模拟试验中, 将试验场实测载荷谱编制成可行有效的试验用载荷谱是十分关键的。提出一套整车室内道路模 拟试验用载荷谱的编制方法, 包括试验场实测载荷谱的选取和试验用载荷谱的加速。对于试验场实测载荷谱的选取, 传统方 法大多基于载荷谱的损伤而选取中值损伤谱。从载荷谱的数值统计特征出发, 提出一种基于各路段最小标准差谱组合的试验 场实测载荷谱选取新方法, 并阐述最小标准差组合谱(Concatenation of multi-sections minimum standard deviation spectra, CMSD)的构造方法与流程。以试验场实测载荷谱为基础构造出 CMSD, 损伤分析结果表明 CMSD 近似为均值损伤谱, 具有 一定的代表性。对于试验用载荷谱的加速, 采用时域信号压缩的方法对 CMSD 进行加速处理, 生成加速谱。以加速谱为目 标谱进行整车耐久性试验, 试验中出现了与汽车试验场一致的失效模式, 验证了该套方法的可行性。该套方法可为其他车型 的整车室内道路模拟试验用载荷谱的编制提供一定的参考。

关键词：室内道路模拟试验；载荷谱选取；最小标准差组合谱；时域信号压缩

中图分类号: U461

\section{Research on Spectrum Development Methodology for Vehicle Indoor Road Simulation Test}

\section{YU Jiawei $^{1}$ ZHENG Songlin ${ }^{1,2}$ ZHAO Lihui ${ }^{1,2}$ ZHAO Zishun ${ }^{3}$}

(1. College of Mechanical Engineering, University of Shanghai for Science and Technology, Shanghai 200093;

2. Machinery Industry Key Laboratory for Mechanical Strength \& Reliability Evaluation of Auto Chassis

Components, University of Shanghai for Science and Technology, Shanghai 200093;

3. Zhejiang Geely Automobile Research Institute Co., Ltd., Hangzhou 311228)

\begin{abstract}
Generation of valid and effective test spectra from proving ground recorded loading spectra is critical in the indoor road simulation test. A set of spectrum development methodology is induced, which includes selection of proving ground recorded loading spectra and acceleration of durability test spectra. For the selection of loading spectra, traditional methods are mostly based on spectrum damage. Statistical characteristics of loading spectrum are taken into account, and a new selection methodology, which is based on concatenation of multi-sections minimum standard deviation spectrum(CMSD), is proposed. The generation method of CMSD is also illustrated. Based on proving ground recorded loading spectra, the CMSD is created. Fatigue damage analyses results have shown that CMSD is equal to mean damage spectra. For the acceleration of test spectrum, time domain signal compression method is applied to develop accelerated spectrum. The accelerated spectrum is applied as the target spectrum, and vehicle durability test is conducted. The fatigue models, which are the same as those of proving ground test, have occurred during the test. The validity of this set of methodology is verified, and it can be a reference for other vehicle indoor road simulation tests.
\end{abstract}

Key words: indoor road simulation test; selection of loading spectra; concatenation of multi-sections minimum standard deviation spectrum(CMSD); time domain signal compression

\section{0 前言}

整车耐久性试验作为评价整车性能的指标之

* 国家高技术研究发展计划(863 计划, 2012AA110701)、国家自然科学 基金(51375313)和上海市科委基础研究重点(13JC1408500)资助项目。 20141023 收到初稿, 20150319 收到修改稿
一越来越受到业界重视。室内道路模拟试验因其可 以较好地再现车辆所承受的道路载荷, 可以昼夜不 间断地连续进行，而且可以对试验用载荷谱进行编 辑处理，缩短试验时间，具有较高的试验效率，从 而被广泛应用 ${ }^{[1-2]}$ 。

试验场采集到的载荷谱会受到驾驶员操作习 
惯、外界环境等随机因素的干扰, 即使是同一个驾 驶员, 按照试验规范行驶过同一路段, 所获得的载 荷谱也是不同的 ${ }^{[3]}$ 。因此, 从多次采集的载荷谱中, 选取最具代表性的载荷谱十分重要。张觉慧等 ${ }^{[4]}$ 提 出运用疲劳统计学母体存活率估计量和矢量模相结 合的基本数学方法, 建立多参数条件下选取存活率 为 $50 \%$ 载荷谱样本的法则。张福泽 ${ }^{[5]}$ 在研究飞机载 荷谱的编制方法时, 提出一种以代表每种实测科目 的中值寿命(或损伤)的起落进行编谱的方法。阎楚 良等 ${ }^{[6]}$ 借助于当量寿命概率分布, 在满足高置信度 ( $90 \%$ 以上) 条件下, 提出了中值随机疲劳载荷谱的编 制原理。以上载荷谱选取方法都是从载荷谱所造成 的损伤这一角度提出的。考虑到试验场的各种特征 强化路面的路况十分恶劣, 在选取载荷谱时, 应选 取最为稳定的, 受到驾驶员和外界随机因素干扰最 小的载荷谱, 这种载荷谱能真实地反映试验场的路 面特征。基于这一角度, 本文从载荷谱自身的数值 统计特征出发, 提出了一种基于各路段最小标准差 谱组合的试验场实测道路谱的选取方法。

以试验场实测载荷谱为基础, 本文首先统计并 识别出每个路段的最小标准差谱对应圈次, 再按照 试验场行驶的先后顺序构造成最小标准差组合谱 (Concatenation of multi-sections minimum standard deviation spectra, CMSD)。之后采用时域信号压缩的 加速处理方法, 对 CMSD 进行编辑处理, 生成加速 谱。采用该加速谱作为目标谱进行迭代, 生成耐久 性试验加载谱并进行整车耐久性试验。试验过程中 出现了与汽车试验场一致的失效模式, 验证了本文 提出的载荷谱编制方法的正确性。

\section{1 道路载荷谱采集}

试验载荷谱采自于襄焚汽车试验场的综合坏 路, 综合坏路包含多种特征强化路面, 如共振路、 蛇形鹅卵石路、比利时路等。本次试验按满载工况 共采集了 9 次综合坏路道路载荷谱, 采样频率为 512 $\mathrm{Hz}$ 。采集信号的类型及通道数分配如表 1 所示。轮 心六分力信号的采集如图 1 所示, 部分底盘零部件 危险部位的应变信号的采集如图 2 所示。

\section{表 1 采集信号的类型及通道数分配}

\begin{tabular}{ccc}
\hline 信号类型 & 数量 & 信号采集对象 \\
\hline 六分力 & 28 & 轮心六分力及轮速 \\
加速度 & 38 & 车身、轮心、发动机悬置加速度 \\
位移 & 4 & 轮心相对车身跳动 \\
应变 & 87 & 车身及底盘零部件危险部位 \\
\hline
\end{tabular}

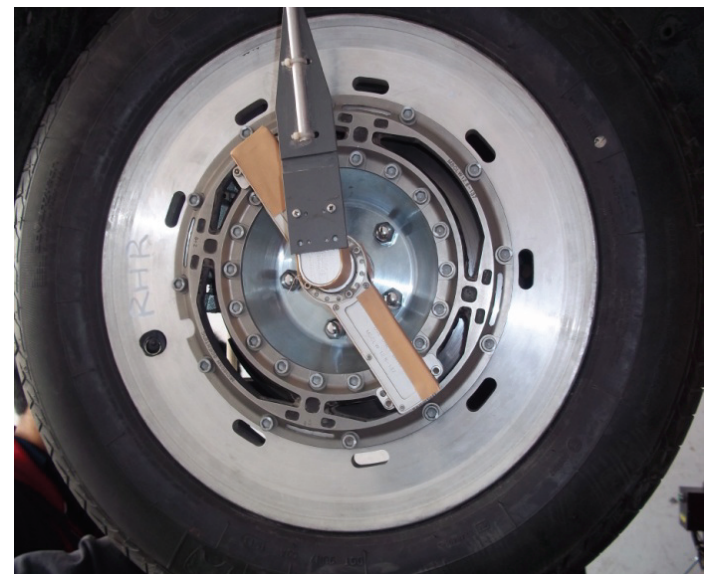

图 1 轮心六分力信号采集

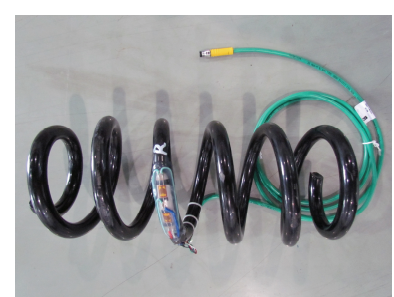

(a) 右后弹簧

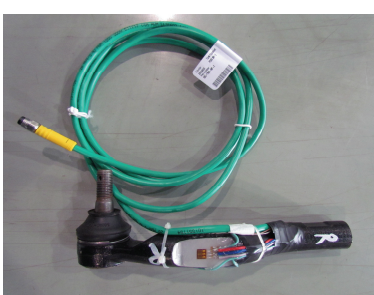

(c) 前右拉杆

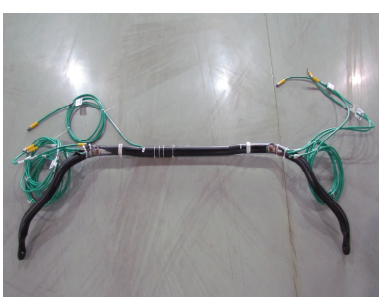

(b) 前稳定杆

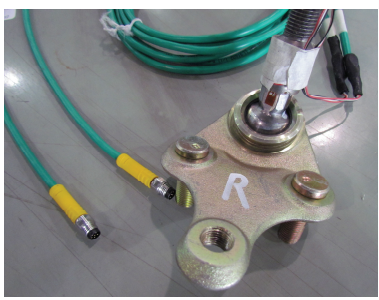

(d) 前右球头
图 2 部分底盘零部件危险部位的应变信号的采集

\section{2 最小标准差组合谱}

同一个司机在重复测量过程中的驾驶方式会 有不同, 即使是同一路段, 同一个驾驶员操作, 所 采集到的信号之间也会存在细微的差异, 这就需要 按照一定的原则从多次采集的信号中篎选出所需要 的信号。标准差可用来衡量随机信号的离散程度, 标准差越小的信号越稳定。因此, 为降低随机因素 的干扰, 本文选取出每种路段的最小标准差谱对应 圈次, 再按试车场行驶的先后顺序构造成 CMSD, 将其作为后续的耐久性试验的目标谱。

\subsection{CMSD 的构造方法}

为构造 CMSD, 首先要统计出每个路段的最小 标准差谱对应圈次, 再将各个路段的对应圈次按试 验场行驶的先后顺序连接成 CMSD。考虑到每个路 段都采集了相同数量的应变通道信号, 并且每个应 变通道都进行了 9 次信号采集。对于某一应变通道 来说, 这 9 次采集信号中必有一次采集信号的标准 差值最小, 对应为该应变通道的最小标准差采集圈 
次。理想的情况是所有应变通道的最小标准差采集 圈次均相同, 然而实际情况却不然。因此, 为统计 每个路段的最小标准差谱对应圈次, 本文采用从多 通道最小标准差采集圈次的汇总结果中选取众数的 方法。具体过程如下。

（1）分离出某一路段的载荷-时间历程, 计算出 该路段每个应变通道的每次采集信号的标准差值, 再将同一应变通道的 9 次采集信号的标准差值按由 小到大的顺序排列, 选取出前三位的采集圈次, 如 式(1)所示

$$
\begin{gathered}
C_{1}=\left\{C_{1 X_{1}}, C_{1 X_{2}}, C_{1 X_{3}} \mid X_{1}, X_{2}, X_{3} \in 1,2, \cdots, 9\right\} \\
C_{2}=\left\{C_{2 X_{1}}, C_{2 X_{2}}, C_{2 X_{3}} \mid X_{1}, X_{2}, X_{3} \in 1,2, \cdots, 9\right\} \\
\vdots \\
C_{n}=\left\{C_{n X_{1}}, C_{n X_{2}}, C_{n X_{3}} \mid X_{1}, X_{2}, X_{3} \in 1,2, \cdots, 9\right\}
\end{gathered}
$$

式中, 下标 $n$ 代表第 $n$ 个应变通道; $X_{1} 、 X_{2} 、 X_{3}$ 代 表某个应变通道的标准差最小的前三次采集; $C_{n}$ 代 表第 $n$ 个应变通道的标准差最小的前三次采集的 集合。

（2）再将该路段所有应变通道的前三位采集圈 次汇总, 构造成集合 $M$, 如式(2)所示

$$
M=\left\{C_{1}, C_{2}, \cdots, C_{n}\right\}
$$

式中, 集合 $M$ 即为多通道最小标准差采集圈次的 汇总。

(3) 从 $M$ 中提取出众数, 该众数对应的采集圈 次既为该路段的最小标准差谱对应圈次。

\section{2 参考信号的确定}

为避免计算量过于繁重, 本文从 87 个应变信 号中选取出少量信号作为参考信号。具体的实现方 法是以兼顾损伤最大和整车损伤分布为原则, 识别 出综合坏路整个循环中损伤最大的几个应变通道, 以这些应变点的信号作为参考信号, 进而选取每个 路段的最小标准差谱对应圈次。在损伤计算时, 由 于此处的目的仅仅为识别出各个信号的相对损伤 值, 没有必要采用精确的零部件 $S-N$ 曲线来计算精 准的损伤值, 因此根据试验车辆各主要零部件所采 用材料的力学性能, 选择 nCode 软件中自带的标准 材料 SAE1035_169 的 $S-N$ 曲线(材料抗拉强度为 550 $\mathrm{MPa}$, 屈服强度为 $410 \mathrm{MPa}, S-N$ 曲线斜率 $b=$ -0.0872 , 曲线拐点对应疲劳寿命为 $10^{6}$ 循环)来计 算各个应变通道的伪损伤值。

计算出各个应变通道的 9 次采集的损伤平均 值, 发现损伤较大的应变通道主要集中在表 2 中所 列出的前 11 个通道。上述应变通道不包含车身上的 测点, 考虑到选取的参考通道应代表整车的损伤分
布, 因此增加车身上的第 124 通道(左前减振器上塔 座)作为参考通道。综合考虑损伤最大和整车损伤分 布, 选取的 12 个参考应变通道如表 2 所示(为方便 比较, 损伤值取为对数)。

表 212 个参考应变通道 (按损伤从大到小排序)

\begin{tabular}{cccc}
\hline 序号 & 通道号 & 对应位置 & $\begin{array}{c}9 \text { 次采集的损伤平均值 } \\
\left(\lg d_{\text {MEAN }}\right)\end{array}$ \\
\hline 1 & 35 & 右前弹簧应变 & -3.7029 \\
2 & 34 & 左前弹簧应变 & -3.8694 \\
3 & 157 & 左前下摆臂球接头载荷 $(y$ 向 $)$ & -4.0238 \\
4 & 37 & 右后弹簧应变 & -4.1125 \\
5 & 156 & 左前下摆臂球接头载荷 $(x$ 向 $)$ & -4.3362 \\
6 & 36 & 左后弹簧应变 & -4.4689 \\
7 & 73 & 右前下摆臂球接头载荷 $(y$ 向 $)$ & -4.5306 \\
8 & 72 & 右前下摆臂球接头载荷 $(x$ 向 $)$ & -4.9413 \\
9 & 45 & 石上臂上下面圆弧处 $(1$ 号点 & -5.0668 \\
10 & 74 & 平行圆弧 & -5 \\
11 & 43 & 右转向横拉杆(轴向 $)$ & -5.3891 \\
12 & 124 & 前稳定杆(轴向 $\left.45^{\circ}\right)$ & -5.5149 \\
\hline
\end{tabular}

\subsection{CMSD 的构造}

按第 2.1 节所提出的方法, 汇总每种路段 12 个 参考通道的标准差最小的前三次采集圈次，提取出 众数, 该众数对应的采集圈次即为该路段的最小标 准差谱对应圈次, 统计结果如表 3 所示, 由于篇幅 所限, 本文只列出前 11 个路段的汇总结果。将每个 路段最小标准差谱对应的载荷一时间历程按试车场 行驶的先后顺序进行连接, 得到综合坏路的 CMSD 如图 3 所示。

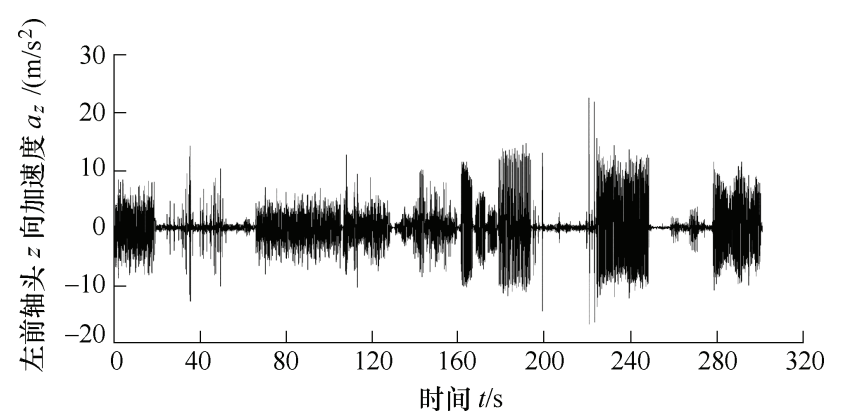

图 3 综合坏路 $\mathrm{CMSD}$ (左前轴头 $z$ 向加速度)

\subsection{CMSD 的分析与验证}

从数值统计上的意义来说, 所构造的 CMSD 是 最稳定的, 受驾驶员和外界随机因素影响最小的载 荷谱。CMSD 的标准差最小, 其损伤值是否为最小 这一问题还有待确定。从理论上来分析, 标准差是 基于载荷谱中离散的数据点计算而得来的, 而损伤 是基于三点或四点雨流循环计数法则从载荷谱中抽 取循环, 再按照疲劳累积损伤理论(线性累积损伤理 论或非线性累积损伤理论)计算得来的 ${ }^{[7]}$, 二者并无 内在的联系。为说明 CMSD 的损伤值特征, 本文计 
算了 CMSD 中所有应变通道的损伤值, 并与 9 次采 本文只列出表 3 中的 12 个参考应变通道的损伤对比 集的相对应通道的损伤平均值对比。由于篇幅所限，

结果，如表 4 所示。

表 3 各路段参考应变通道标准差最小的前三次采集圈次汇总

\begin{tabular}{|c|c|c|c|c|c|c|c|c|c|c|c|}
\hline 通道 & 形鹅卵石路 & 破损路 & 比利时路 & 砂石路 & 搓板路 & 共振路 3 & 过铁轨路 & 坑洼路 & 共振路 2 & 路缝接合路 & 共振路 1 \\
\hline 34 & $2,8,6$ & $4,6,1$ & $1,3,7$ & $9,3,4$ & $1,2,7$ & $6,5,7$ & $4,1,9$ & $1,3,9$ & $1,4,6$ & $5,7,1$ & $1,8,4$ \\
\hline 35 & $5,6,9$ & $1,6,3$ & $1,3,7$ & $3,7,5$ & $1,2,4$ & $5,3,6$ & $4,6,2$ & $1,5,2$ & $1,4,6$ & $3,1,7$ & $1,4,8$ \\
\hline 36 & $5,6,9$ & $8,3,4$ & $1,7,3$ & $4,7,1$ & $1,7,2$ & $7,5,6$ & $4,5,3$ & $1,7,2$ & $1,6,4$ & $8,3,5$ & $8,1,4$ \\
\hline 37 & $2,8,3$ & $1,6,7$ & $1,3,4$ & $1,4,8$ & $1,2,7$ & $1,4,7$ & $3,2,5$ & $9,1,2$ & $1,4,7$ & $3,5,8$ & $1,8,4$ \\
\hline 43 & $2,5,6$ & $6,1,3$ & $3,1,6$ & $1,2,3$ & $2,1,7$ & $1,6,3$ & $3,8,6$ & $7,1,2$ & $1,4,6$ & $9,2,1$ & $1,8,4$ \\
\hline 45 & $2,9,5$ & $6,1,3$ & $3,8,7$ & $1,5,3$ & $1,2,4$ & $1,6,8$ & $3,6,1$ & $1,4,9$ & $9,8,1$ & $9,3,7$ & $8,1,4$ \\
\hline 72 & $7,3,6$ & $6,7,8$ & $9,8,7$ & $1,7,3$ & $2,6,7$ & $3,6,8$ & $4,6,5$ & $5,9,6$ & $2,3,8$ & $3,5,9$ & $7,8,1$ \\
\hline 73 & $6,3,9$ & $6,3,7$ & $9,7,8$ & $5,7,2$ & $1,7,2$ & $6,8,7$ & $4,5,6$ & $6,5,7$ & $1,7,6$ & $3,7,5$ & $8,9,7$ \\
\hline 74 & $6,5,2$ & $6,7,3$ & $9,1,4$ & $1,2,7$ & $2,1,7$ & $6,5,4$ & $4,5,7$ & $1,6,4$ & $6,4,1$ & $5,7,9$ & $3,8,7$ \\
\hline 124 & $6,8,2$ & $4,6,1$ & $1,7,9$ & $1,7,8$ & $1,2,6$ & $8,6,1$ & $9,6,4$ & $2,1,3$ & $7,9,4$ & $7,5,8$ & $8,3,1$ \\
\hline 156 & $2,6,4$ & $4,6,3$ & $1,3,6$ & $1,3,7$ & $2,1,8$ & $8,7,6$ & $4,7,6$ & $8,6,9$ & $6,7,1$ & $3,5,8$ & $9,7,3$ \\
\hline 157 & $1,2,6$ & $4,8,3$ & $1,7,3$ & $5,3,1$ & $1,2,7$ & $8,6,9$ & $4,7,5$ & $5,8,4$ & $4,8,6$ & $4,5,8$ & $9,8,7$ \\
\hline 统计结果 & 6 & 6 & 1 & 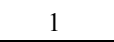 & 1 & 6 & 4 & 1 & 1 & 3 & 8 \\
\hline
\end{tabular}

表 4 CMSD 的损伤值与 9 次采集的损伤平均值对比 (按损伤由大到小排序)

\begin{tabular}{|c|c|c|c|c|c|}
\hline 序号 & 通道号 & 对应位置 & 损伤平均值 $\left(\lg d_{\text {MEAN }}\right)$ & CMSD 损伤值 $\left(\lg d_{\text {CMSD }}\right)$ & 比例 \\
\hline 1 & 35 & 右前弹簧应变 & -3.7029 & -3.5894 & 0.9693 \\
\hline 2 & 34 & 左前弹簧应变 & -3.8694 & -3.6671 & 0.9477 \\
\hline 3 & 157 & 左前下摆臂球接头载荷 $(y$ 向) & -4.0238 & -4.2411 & 1.0540 \\
\hline 4 & 37 & 右后弹簧应变 & -4.1125 & -4.0928 & 0.9952 \\
\hline 5 & 156 & 左前下摆臂球接头载荷 $(x$ 向 $)$ & -4.3362 & -4.3196 & 0.9962 \\
\hline 6 & 36 & 左后弹簧应变 & -4.4689 & -4.3938 & 0.9832 \\
\hline 7 & 73 & 右前下摆臂球接头载荷 $(y$ 向) & -4.5306 & -4.6008 & 1.0155 \\
\hline 8 & 72 & 右前下摆臂球接头载荷 $(x$ 向) & -4.9413 & -4.8857 & 0.9888 \\
\hline 9 & 45 & 右上臂上下面圆弧处 & -5.0668 & -4.7995 & 0.9473 \\
\hline 10 & 74 & 右转向横拉杆(轴向) & -5.3891 & -5.3310 & 0.9892 \\
\hline 11 & 43 & 前稳定杆(轴向 45) & -5.5149 & -5.6173 & 1.0186 \\
\hline 12 & 124 & 左前减振器上塔座 & -12.7284 & -13.8871 & 1.0910 \\
\hline
\end{tabular}

表 4 中，第 124 通道的损伤值很小，量级为 $10^{-12}$, 载荷谱中的略微波动会对其损伤值产生较大 影响，且考虑到某些具有很长寿命的结构件，其疲 劳寿命在 $N_{\mathrm{f}}=10^{8} \sim 10^{10}$ 周的范围内 ${ }^{[8]}$ 。从损伤的角 度来说其不具备参考价值, 因此舍去第 124 通道的 对比结果。由其余 11 个参考应变通道的损伤对比结 果可得出, 比值的均值为 0.9914 , 近似为 1 。而考 虑所有应变通道(舍去损伤值较小的、量级低于 $10^{-10}$ 的通道)的损伤对比结果, 如图 4 所示, 为所有应变 通道的 9 次采集的损伤均值与对应通道的 CMSD 损 伤值的散点图(双对数坐标), 拟合后的公式为

$$
y=1.03415 x+0.18828
$$

可知 CMSD 中各应变通道的损伤值相对于各 自的损伤均值上下轻微波动, 而考虑多通道的综合 效果, CMSD 近似为均值损伤谱, 具有一定的代 表性。

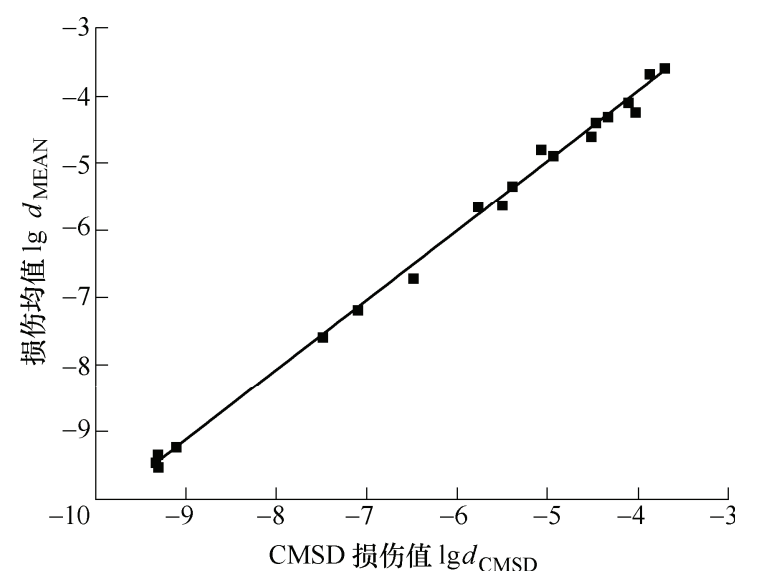

图 4 所有应变通道(损伤量级高于 $10^{-10}$ ) 9 次采集的损伤 均值与对应通道的 CMSD 损伤值的散点图

\section{3 试验用载荷谱的时域信号压缩}

以 CMSD 作为试验目标谱。对试验目标谱进行 编辑, 主要包括目标谱的预处理和目标谱的加速处 
理。目标谱的预处理主要包括去除信号漂移、删除 尖峰异常值等工作。载荷谱的加速处理可从时域、 幅值域和频域三个方面来实现 ${ }^{[9]}$ 。幅值域和频域这 两种编辑方法会造成载荷的加载顺序和多通道之间 相位关系的丢失 ${ }^{[10]}$, 无法复现试验场真实的载荷时间历程, 因此无法满足室内道路模拟试验的基本 要求。为了保留原始谱的加载顺序关系, 保证多通 道之间的相位关系和原始谱的频率特性, 本文采用 时域信号压缩的编辑处理方法, 即在载荷-时间历程 中删除低幅值载荷对应的时间历程, 在实现加速试 验的同时保证了道路模拟试验的基本要求。

\section{1 低幅值载荷删除國值的确定}

在载荷谱的时域编辑处理中, 目前大多数的做 法是删除材料疲劳极限 $50 \%$ 以下的小载荷 ${ }^{[11]}$, 或删 除最大载荷的 $10 \%$ 以下的小载荷 ${ }^{[12]}$, 认为这样做不 会改变试样的疲劳损伤。依据作者所在课题组多年 来的研究成果 ${ }^{[13-15]}, 0.65 \sigma_{-1}$ 以下的载荷对零件的疲 劳无任何效果 ${ }^{[16-17]}$, 可以认定为无效载荷, 在载荷 谱的编辑处理时可以予以删除。

首先估算零部件的 $\sigma_{-1 \mathrm{~d}}$ (零部件的对称弯曲疲劳 极限)。试验车辆底盘大部分零部件所用材料的抗拉 强度 $\sigma_{\mathrm{b}}=550 \mathrm{MPa}$ ，估算得到材料的对称弯曲疲劳极 限为 $\left(10^{7}\right.$ 循环 $)$

$$
\sigma_{-1}=0.5 \sigma_{\mathrm{b}}=275 \mathrm{MPa}
$$

对 $\sigma_{-1}$ 进行适当的修正，公式为

$$
\sigma_{-1 \mathrm{~d}}=\sigma_{-1} C_{\mathrm{L}} C_{\mathrm{D}} C_{\mathrm{S}} C_{\mathrm{R}} / K_{\mathrm{f}}
$$

式中, $C_{\mathrm{L}}$ 为载荷系数; $C_{\mathrm{D}}$ 为尺寸系数; $C_{\mathrm{S}}$ 为表面 质量系数; $C_{\mathrm{R}}$ 为可靠性水平系数; $K_{\mathrm{f}}$ 为疲劳缺口系 数。参考文献 [3], 各项系数的取值为 $C_{\mathrm{L}}=0.75$, $C_{\mathrm{D}}=0.9, C_{\mathrm{S}}=0.7, C_{\mathrm{R}}=1, K_{\mathrm{f}}=1.5$; 估算得到的 $\sigma_{-1 \mathrm{~d}}=87$ $\mathrm{MPa}$ 。计算得到低幅值载荷的删除阈值

$$
0.65 \sigma_{-1 \mathrm{~d}}=57 \mathrm{MPa}
$$

确定低幅值载荷的删除阈值为 $57 \mathrm{MPa}$ 。为方便 进行删除低幅值载荷的编辑处理, 同时考虑到汽车 零部件的疲劳失效模式大多为高周疲劳, 应变主要 为弹性应变, 将应力除以弹性模量 $E$ 转换为相应的 应变为 $269 \times 10^{-6}$ 。

\section{2 时域信号压缩前后的载荷-时间历程对比}

台架试验的目标信号选为四个车轮轴头的垂 向加速度信号, 本文没有直接计算并确定出加速度 信号的删除阈值, 而是以多通道应变信号为参照, 在删除多通道应变信号的小载荷 $\left( \pm 269 \times 10^{-6}\right)$ 对应的 载荷-时间历程时, 同时删除加速度信号对应的载荷时间历程, 从而实现加速度信号的编辑处理。需要 说明的是, 只有在所有应变通道对应的载荷-时间历
程均为阈值以下的小载荷时, 该载荷-时间历程才会 被删除。编辑后的加速谱与原始谱的载荷-时间历程 对比如图 5 所示。经删除无效载荷后, 加速谱缩短 为 $135 \mathrm{~s}$, 为原始谱的 $45 \%$, 加速效果较为明显。

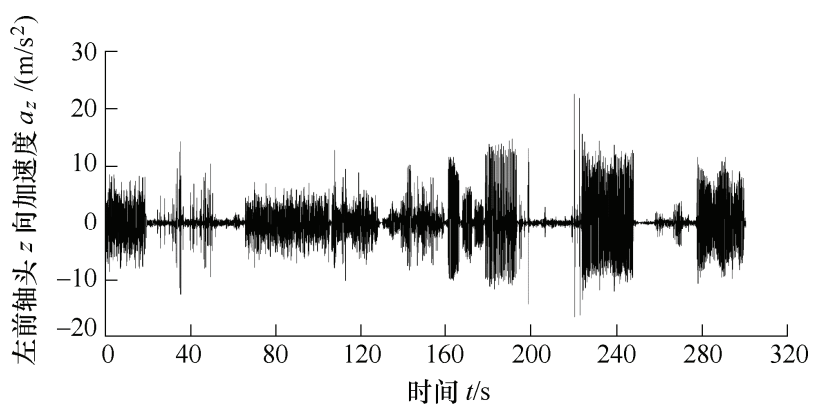

（a）原始谱载荷-时间历程

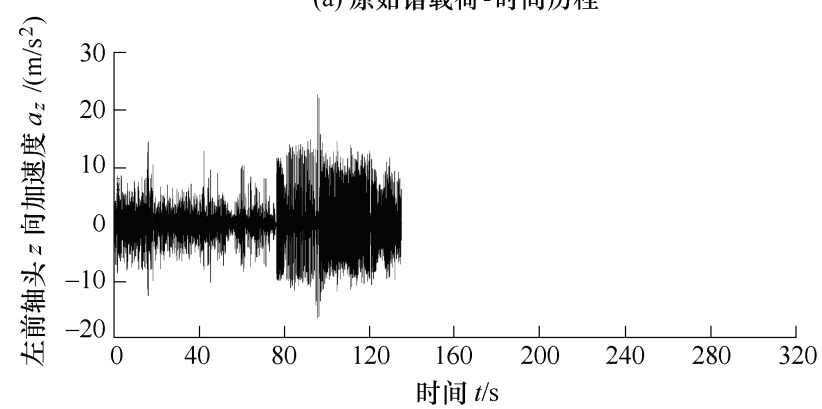

(b) 加速谱载荷-时间历程

图 5 原始谱与加速谱时间长度对比

\section{4 室内道路模拟试验验证}

对于整车及其零部件耐久性试验用载荷谱的 编制方法, 所要保证的重要原则是试验中出现的失 效部位、失效模式以及寿命分布应与道路试验相一 致 ${ }^{[18]}$ 。以编制后生成的加速谱作为试验的目标信 号, 经过迭代获得四立柱试验台四个液压缸位移的 驱动信号。在迭代过程中, 以四轮轴头的垂向加速 度信号作为迭代目标, 将误差临界值规定为 $10 \%$ 。 通过实时的观测，一步步地进行迭代，力求迭代误 差达到最小。当迭代误差已经达到允许值, 进一步 迭代出现发散现象时，则选取前一次迭代作为有效 迭代次数。

进行室内耐久性试验时, 需要时刻观察试件是 否出现失效现象, 记录失效现象出现的时间。在本 次试验中, 出现了以下部位的失效情况: $134 \mathrm{~h} 35$ $\min$ 时, 发现后桥稳定杆卡䈨撕裂, 如图 6 所示。 试验结束后, 发现以下部位的失效情况：右后轮罩 开裂, 如图 7 所示; 左后轮罩连接板开裂, 如图 8 所示。这些失效现象出现的部位及失效模式均与试 验场道路试验相一致。然而, 由于试验对象为整车, 样本有限, 且试验费用昂贵, 因此很难验证失效模 式的寿命分布是否与道路试验一致。以上试验结果 
表明, 采用该套编谱方法建立的整车室内道路模拟 试验可代替试验场道路试验来考核整车的耐久性, 在一定程度上验证了该套编谱方法的可行性。该套 方法可为其他车型的整车室内道路模拟试验用载荷 谱的编制提供一定的参考。

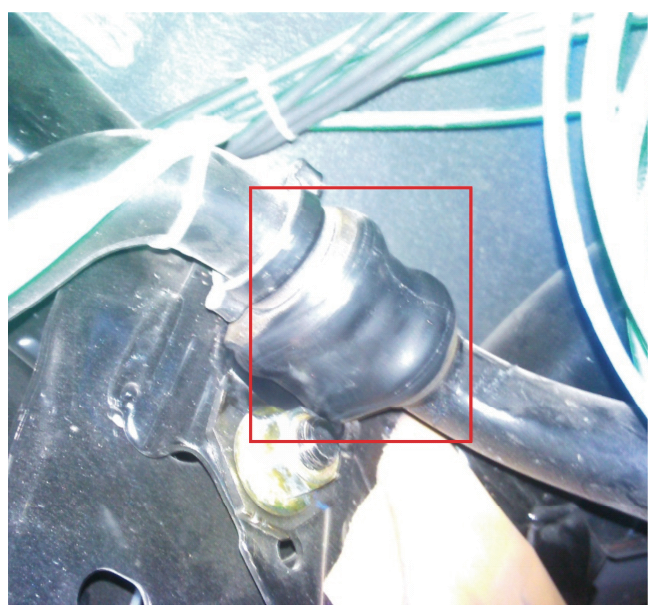

图 6 后桥稳定杆卡箱撕裂

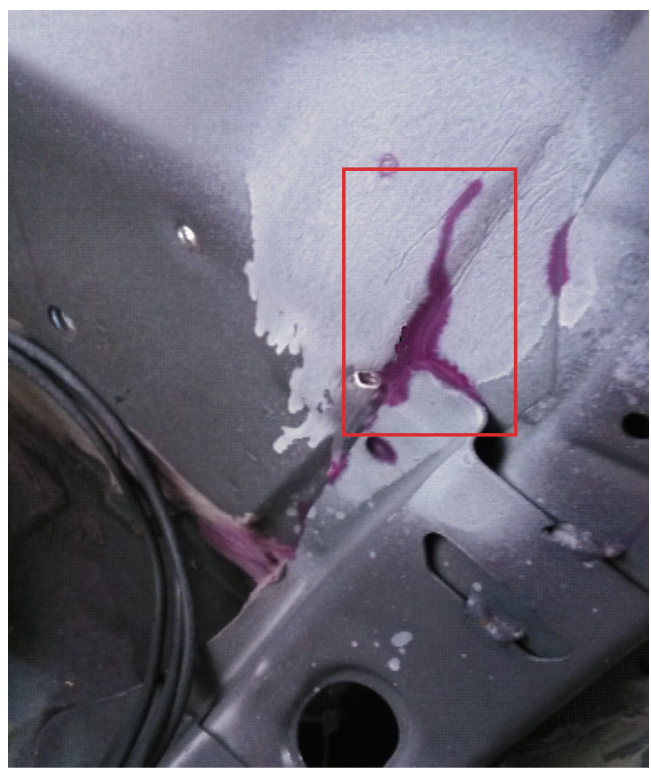

图 7 右后轮罩开裂

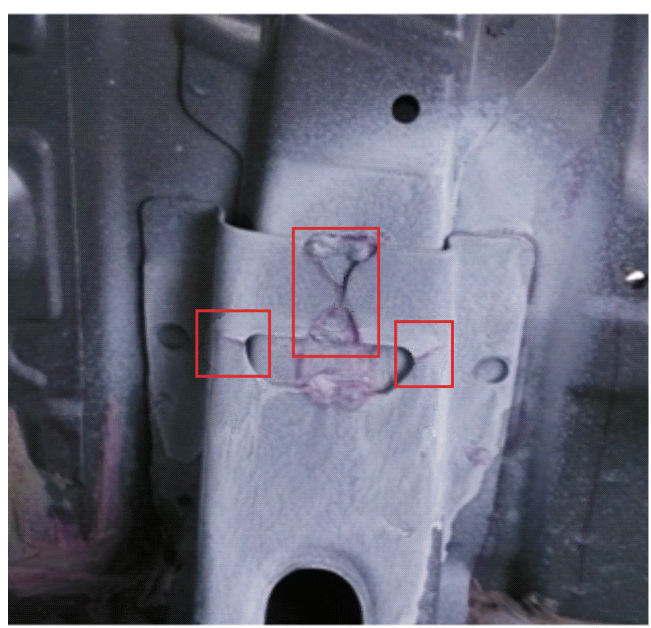

图 8 左后轮罩连接板开裂

\section{5 结论}

本文提出了一套整车室内道路模拟试验用载 荷谱的编制方法。

（1）基于载荷谱的数值统计特征，提出了基于 各路段最小标准差谱组合的载荷谱选取新方法, 阐 述了 CMSD 的构造方法及流程; 以实测载荷谱为基 础, 构造出了 CMSD。损伤分析结果表明 CMSD 近 似于均值损伤谱，具有一定的代表性。

（2）考虑室内道路模拟试验对载荷的加载顺序 关系, 以及多通道载荷之间的相位关系的基本要求, 采取了时域信号压缩的方法对 CMSD 进行了加速 处理, 生成了加速谱, 加速效果较为明显。

（3）为验证所采用的载荷谱编制方法的正确 性, 以加速谱为目标谱, 生成四立柱试验台四个液 压缸位移驱动信号并进行整车耐久性试验, 试验中 出现了与汽车试验场一致的失效模式, 从而在一定 程度上验证了该套方法的可行性, 该套方法可为其 他车型的整车室内道路模拟试验用载荷谱的编制提 供一定的参考。

\section{参 考 文 献}

[1] 丛楠, 尚建忠, 任炎睎. 基于滑动分块 Bootstrap 方法 的非结构道路谱 $\alpha$-稳定分布参数区间估计与重构 [J]. 机械工程学报，2013，49(4): 106-113.

CONG Nan, SHANG Jianzhong, REN Yanxi. Unstructured road apectrum $\alpha$-stable distribution parameters interval estimation and reconstruction based on moving block bootstrap method[J]. Journal of Mechanical Engineering, 2013, 49(4): 106-113.

[2] 钱立军, 吴道俊, 杨年昫, 等. 基于室内道路模拟技术 的整车加速谱耐久性试验的研究[J]. 汽车工程, 2011, 33(2): 91-96.

QIAN Lijun, WU Daojun, YANG Nianjiong, et al. A research on vehicle accelerated durability test based on indoor road simulation technology[J]. Automotive Engineering, 2011, 33(2): 91-96.

[3] LEE Y L, PAN J, HATHAWAY R B, et al. Fatigue testing and analysis(Theory and Practice)[M]. Burlington : Elsevier Butterworth-Heinemann, 2005.

[4] 张觉慧, 金峰, 余卓平. 道路模拟试验用载荷谱样本选 择方法[J]. 汽车工程, 2004, 26(2): 220-223.

ZHANG Juehui, JIN Feng, YU Zhuoping. A method of selecting load spectra for road simulation Test[J]. Automotive Engineering, 2004, 26(2): 220-223. 
[5] 张福泽. 飞机载荷谱编制的新方法研究 [J]. 航空学报, 1998, 19(5): 518-524.

ZHANG Fuze. New method of drawing up aircraft load spectrum[J]. Acta Aeronautica Et Astronautica Sinica, 1998, 19(5): 518-524.

[6] 阎楚良, 高镇同. 飞机高置信度中值随机疲劳载荷谱的 编制原理[J]. 航空学报, 2000, 21(2): 118-123.

YAN Chuliang, GAO Zhentong. Compilation theory of median stochastic fatigue load-spectrum with high confidence level for airplane[J]. Acta Aeronautica Et Astronautica Sinica, 2000, 21(2): 118-123.

[7] MCINNES C H, MEEHAN P A. Equivalence of four-point and three-point rainflow cycle counting algorithms[J]. International Journal of Fatigue, 2008, 30: 547-559.

[8] 郑修麟, 王泓, 滒君辉, 等. 材料疲劳理论与工程应用 [M]. 北京: 科学出版社, 2013.

ZHENG Xiulin, WANG Hong, YAN Junhui, et al. Material fatigue theory and engineering application[M]. Beijing: Science Press, 2013.

[9] 王铁, 赵富强, 张瑞亮, 等. 变速器加速疲劳试验方法 分析[J]. 汽车工程, 2013，35(12): 1078-1083.

WANG Tie, ZHAO Fuqiang, ZHANG Ruiliang, et al. An analysis on accelerated fatigue test method for transmissions[J]. Automotive Engineering, 2013, 35(12): 1078-1083.

[10] HEULER P, KLATSCHKE H. Generation and use of standardized load spectra and load-time histories[J]. International Journal of Fatigue， 2005， 27: 974-990.

[11] HEULER P, SEAGER T. A criterion for omission of variable amplitude loading histories[J]. International Journal of Fatigue, 1986， 8(4): 225-300.

[12] AICHER W, BRANGER J, van DIJK G M, et al. Description of a fighter aircraft loading standard for fatigue evaluation FALSTAFF[R]. Common Report of F+W Emmen, LBF, NLR, IABG, 1976.

[13] 储军, 郑松林, 冯金芝, 等. 基于低幅锻炼载荷的累积 强化效果模型 $[J]$. 机械工程学报, 2011, 47(16): 30-34.
CHU Jun, ZHENG Songlin, FENG Jinzhi, et al. Cumulative strengthening effect model based on the low-amplitude training load[J]. Journal of Mechanical Engineering, 2011, 47(16): 30-34.

[14] ZHENG Songlin, XU Honghui, FENG Jinzhi, et al. Lightweight design of automobile drive shaft based on the characteristics of low amplitude loading strengthening $[\mathrm{J}]$. Chinese Journal of Mechanical Engineering, 2011, 24(6): 1111-1115.

[15] LU Xi, ZHENG Songlin. Strengthening of transmission gear under low-amplitude load[J]. Material Science and Engineering A, 2008, 488: 55-63.

[16] 赵礼辉, 郑松林, 冯金芝. 基于低载强化特性的疲劳寿 命估计方法[J]. 机械工程学报, 2013，49(8): 115-122. ZHAO Lihui, ZHENG Songlin, FENG Jinzhi. Fatigue life prediction based on simplified low-amplitude-load strengthening model[J]. Journal of Mechanical Engineering, 2013, 49(8): 115-122.

[17] 郑松林, 梁国清, 王治瑞, 等. 考虑低幅锻炼载荷的某 轿车摆臂载荷谱编制[J] 机械工程学报, 2014, 50(16): 147-154.

ZHENG Songlin, LIANG Guoqing, WANG Zhirui, et al. Compilation of automotive lower control arm spectrum based on the low-amplitude training load[J]. Journal of Mechanical Engineering, 2014, 50(16): 147-154.

[18] 高云凯, 徐成民, 方剑光. 车身台架疲劳试验程序载荷 谱研究 [J]. 机械工程学报, 2014，50(4): 92-98.

GAO Yunkai, XU Chengmin, FANG Jianguang. Study on the programed load spectrum of the body fatigue bench test[J]. Journal of Mechanical Engineering, 2014, 50(4): 92-98.

作者简介: 于佳伟, 男, 1990 年出生, 博士研究生。主要研究方向为车 辆载荷特性、结构耐久性与可靠性快速评价。

E-mail: jia_wei_yu@yeah.net

郑松林(通信作者), 男, 1958 年出生, 教授, 博士研究生导师。主要研 究方向为车辆载荷特性、结构强度、振动及可靠性评估方法等。

E-mail: songlin_zheng@126.com 